\title{
Filtros de echado recursivos
}

\author{
A. Cabrales-Vargas y S. Chávez-Pérez \\ Facultad de Ingeniería, UNAM e Instituto Mexicano del Petróleo \\ E-mails:metalhead@elsitio.comyschavez@imp.mx \\ (recibido: octubre de 2001; aceptado: febrero de 2002)
}

\begin{abstract}
Resumen
En sismología de exploración, los filtros de echado se utilizan para enfatizar ímágenes del subsuelo, atenuando ruido coherente y otras señales. Pueden aplicarse en los dominios de frecuencia y número de onda $(f-k)$, frecuencia y distancia $(f-x)$, tiempo y número de onda $(t-k)$ o tiempo y distancia $(t-x)$. En el dominio de Fourier suponemos echados constantes. Los filtros de echado recursivos se aplican en el dominio $t-x$, careciendo de esta limitante. Sin embargo, tenemos que recurrir al ensayo y error para determinar sus parámetros óptimos. Los filtros de echado recursivos se basan en filtros de Butterworth de orden uno, añadiendo el número de onda. Su espectro de amplitud es una superficie. Utilizamos la transformada bilineal para digitalizarlos y pasar del dominio $f-k$ al $t-k$. Antitransformando el número de onda obtenemos los filtros en $t-x, y$ los aproximamos con tres coeficientes, generando así una matriz tridiagonal. Para fines de ilustración en ingeniería geofísica, aplicamos estos filtros en un registro de campo somero para atenuar la onda de aire y el ruido aleatorio, y en una sección marina enfatizamos una zona de falla. Ambos ejemplos muestran que estos filtros son útiles y prácticos para enfatizar datos sísmicos. Su uso es más sencillo y económico que el de los filtros de mediana, utilizados actualmente en paquetes comerciales para la industria del petróleo.
\end{abstract}

Descriptores: sismología de exploración, filtrado, dominio de Fourier, filtro de Butterworth, enfatización de imágenes.

\footnotetext{
Abstract

In exploration seismology, dip filters are used to enhance subsoil images by attenuating coherent noise and other signals. They can be applied in frequency-wavenumber $(f-k)$, frequency-distance $(f-x)$, time-wavenumber $(t-k)$ or time distance $(t-k)$ domains. Fourier domain assumes constant dips. Recursive dip filters are applied in $t-x$ domain, as they do not have this limitation. However, we have to determine their optimal parameters by trial and error. Recursive dip filters are based on single order Butterworth filters, by adding the wavenumber. Their amplitude spectrum is a surface. We perform a bilinear transform to digitize the filter and pass from the $f-k$ to the $t-k$ domain. We obtain the $t-x$ domain filter by inverse transforming through wavenumber and by using a three-coefficient approximation (leading to a tridiagonal matrix). For the sake of illustration in geophysical engineering, we apply these filters to a shallow field record, to attenuate the air wave and random noise, and to a marine seismic section to enhance a fault zone. Both examples show that these filters are useful and practical to enhance seismic data. Their use is easier and more economical than median filters, utilized nowadays in commercial software for the oil industry.
} 
DOI: http://dx.doi.org/10.22201/fi.25940732e.2002.03n3.013

\section{Introducción}

El proceso de filtrado en sismología de exploración juega un papel importante para la atenuación de ruido. Normalmente pensamos en un filtro como un artefacto numérico que permite el paso de valores de una banda de frecuencias, mientras nulifica por completo todas las demás. Idealmente, un filtro en el dominio de la transformada de Fourier representa una función caja. Sin embargo, en el dominio del tiempo los filtros ideales son funciones de muestreo (sampling), cuya expresión es del tipo $(\operatorname{sen} \Omega t) /(\Omega t)$, donde $\Omega$ es la frecuencia de corte. Estas funciones se atenúan muy lentamente para su uso práctico, debido a que debemos truncar el filtro, y se requeriría de un gran número de muestras para reconstruir adecuadamente la caja en frecuencias. Por su parte, un filtro cuya respuesta en tiempo decae con rapidez se aleja demasiado de la forma ideal en el dominio de las frecuencias (Claerbout, 1992).

Los eventos que son horizontales en el dominio $x-t$ tienden a reunirse alrededor del eje de la frecuencia en el dominio $f-k$. A medida que su inclinación aumenta, se desplazan hacia el eje del número de onda, semejando un abanico. Por lo tanto, el filtrado en el dominio de la frecuencia y el número de onda resulta sencillo, al menos en lo que se refiere a la selección de la banda de paso del filtro. El filtrado se efectúa transformando los datos de acuerdo con Fourier, suprimiendo la banda de corte deseada y transformando en forma inversa, de manera que la sección "antitransformada" carece, en teoría, de las señales que quedaron fuera de la banda de paso. Sin embargo, filtrar en el dominio de la transformada de Fourier supone que los echados no varían en función de la dimensión que ha sido transformada. Así, es posible utilizar filtros que solamente requieran la transformada de Fourier de una de las dimensiones, pero suponiendo que los echados son invariables con respecto a esta dimensión. La otra alternativa es filtrar en el dominio del tiempo y la distancia.

Un tipo especial de filtrado se denomina "de echado". Su aplicación en sismología de exploración se ha dado en la medida que ha sido necesario atenuar ciertas señales y ruido coherente e incoherente en datos sísmicos, con el fin de enfatizar las imágenes.
Este trabajo tiene como objetivo dar a conocer en términos simples, las ventajas de los filtros de echado recursivos, sus inconvenientes, y ejemplificar su aplicación con datos sísmicos de exploración. Este tipo de filtrado consiste en una adaptación de los filtros de Butterworth, de modo que el espectro de amplitud ya no constituye una curva, sino una superficie alabeada (Figuras 1, 2 y 3). El orden de los filtros en las figuras 1, 2 y 3 es de 10, para visualizar mejor la región de corte. El parámetro $\rho$ es el echado de corte, dado por $\rho=n_{t} / n_{x}$, donde $n_{t}$ es el número de muestras recorridas en $t$ cuando se recorren $n_{x}$ muestras en $x$. El eje de las frecuencias está expresado en términos de $f$. El comportamiento de estos filtros es similar al de los filtros en frecuencias. Cortes realizados para números de onda constantes dan lugar a filtros en frecuencia, y lo mismo se aplica para cortes realizados para frecuencias constantes (Figura 4).

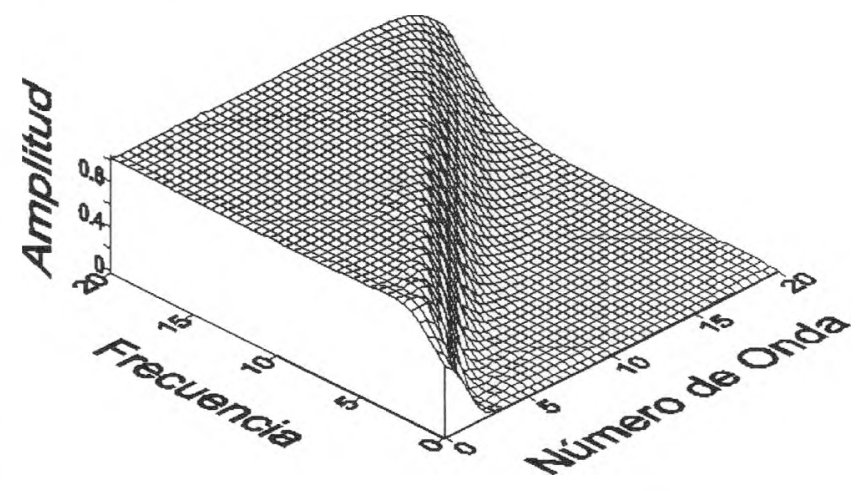

Figura 1. Espectro de amplitud de un filtro de echado recursivo pasabajos, con parámetros $\rho=1$ y $f_{n}=0.5$

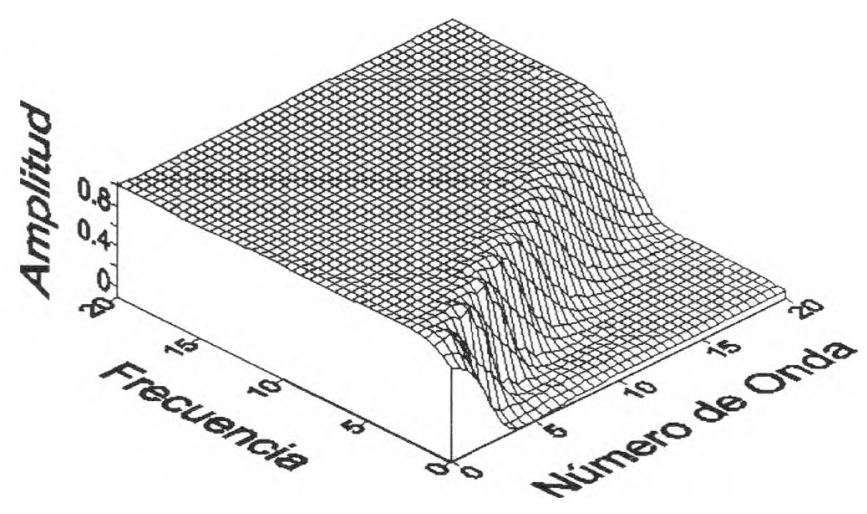

Figura 2. Espectro de amplitud de un filtro de echado recursivo pasabajos, con parámetros $\rho=2$ y $f_{n}=0.5$ 


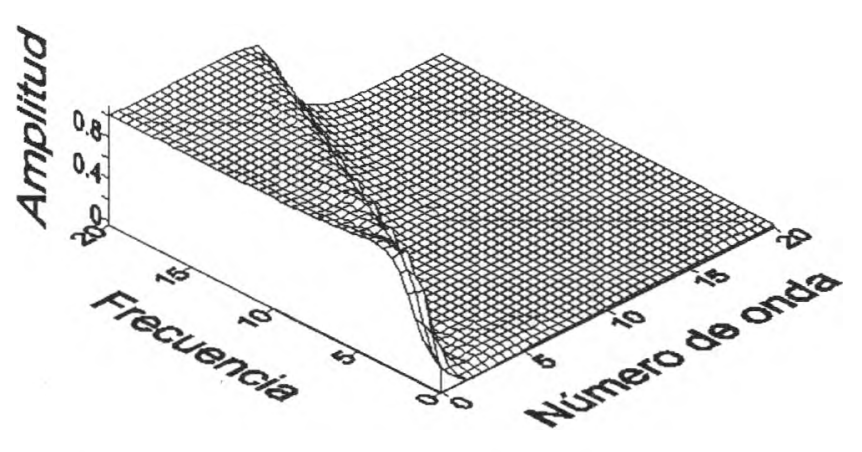

Figura 3. Espectro de amplitud de un filtro de echado recursivo pasabajos, con parámetros $\rho=0.5$ y $f_{n}=0.5$
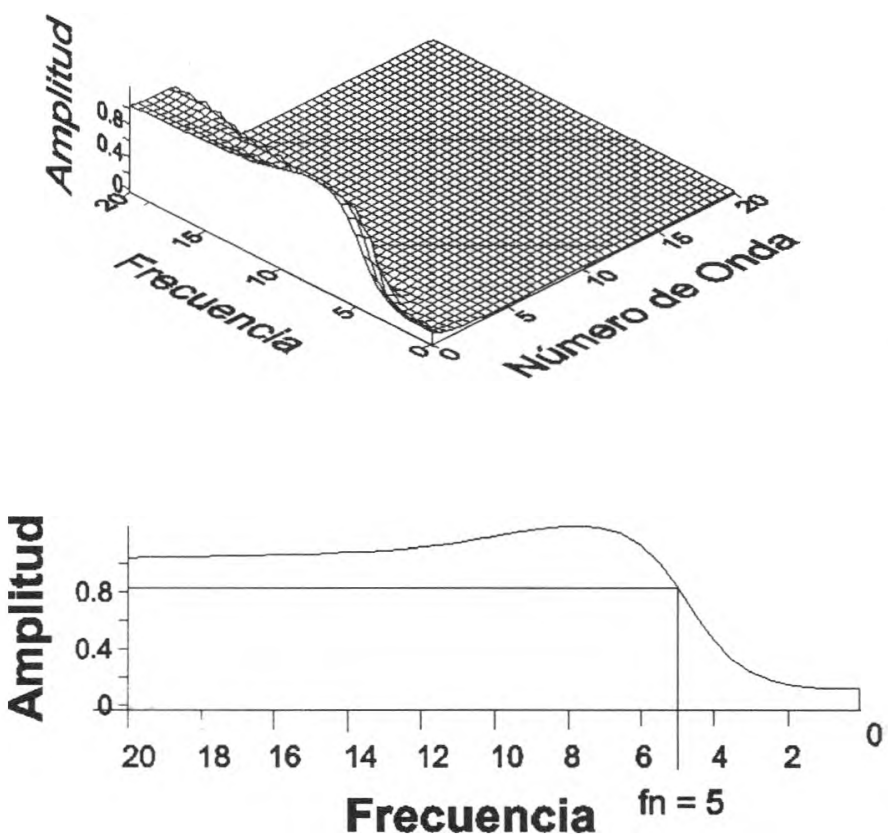

Figura 4. Arriba: Espectro de amplitud de un filtro de echado recursivo, con parámetros $\rho=1$ y $f_{n}=2.5$. Abajo: Corte del filtro para el número de onda igual a cero. Como resultado, obtenemos un filtro pasaaltas, con frecuencia de Nyquist igual a 0.5. El aumento en el valor de $f_{n}$ reduce la banda de paso

Las formas en que operan este tipo de filtros son:

a) En el dominio de la frecuencia y el número de onda $(f-k)$;

b) En el dominio del tiempo y el número de onda $(t-k)$; c) En el dominio de la frecuencia y la distancia $(f-\chi)$;

d) En el dominio del tiempo y la distancia $(t-\chi)$.

Las ventajas que presenta esta última variante provienen de los siguientes hechos:

No es posible tomar en cuenta variaciones del echado con respecto a una dimensión que se transforma de acuerdo con Fourier. En el dominio $f-k$ no suponemos variación alguna. En los dominios donde sólo una de las dimensiones ha sido transformada (e.g., $f-x$ y $t-k$ ) podemos suponer variaciones en la dimensión sin transformar. En el dominio del tiempo y la distancia no existen restricciones en la variación en una u otra dirección, dando lugar a un resultado más realista

El filtrado $f-k$ presenta problemas cuando existe el fenómeno de submuestreo (aliasing) (Spitz, 1993), en el que las amplitudes más allá de un valor de frecuencia denominado de Nyquist se desplazan hacia las frecuencias bajas, adicionándose con las amplitudes que ahí se encuentren. La frecuencia de Nyquist varía en forma inversa con el intervalo de muestreo. Así, una amplitud cuya frecuencia $f_{s}$ es mayor que la frecuencia de Nyquist, $f_{N}$, quedará desplazada a un valor de frecuencia $f_{a}$ dado por

$$
f_{a}=\left|2 m f_{\mathrm{N}}-f_{\mathrm{s}}\right|
$$

donde $m$ es un valor entero tal que se cumpla que $f_{a}<f_{N}$ (Yilmaz, 2000). En los filtros bidimensionales, el submuestreo puede afectar una o ambas dimensiones.

Existen también inconvenientes con los filtros de echado recursivos, pues no podemos visualizar la banda de paso del filtro con la misma facilidad que en los filtros $f-k$. Además, resulta difícil en la práctica establecer adecuadamente el valor del echado de corte. Esto ocurre porque el ritmo de atenuación es muy suave, de modo que el filtro generalmente debe programarse para rechazar un intervalo de valores de echado mayor al que teóricamente debería ser. La razón de ello es la aproximación por medio de un polo. Por otro lado, no existe una versión pasabandas del filtro, como ocurre con los filtros $f-k$. 
DOI: http://dx.doi.org/10.22201/fi.25940732e.2002.03n3.013

\section{Derivación de las expresiones para los filtros de echado}

Los filtros, aunque se apliquen en el dominio del tiempo, del número de onda, o de ambos, resultan más fáciles de elaborar y visualizar en el dominio de la transformada de Fourier bidimensional. En el caso de los filtros unidimensionales de Butterworth en el dominio de la frecuencia, tenemos las siguientes expresiones para los espectros de potencia (Claerbout, 1992)

$$
\begin{aligned}
& |H(\omega)|^{2}=\frac{1}{1+(\omega / \Omega)^{2 n}} \text { Filtro Pasabajas, } \\
& |H(\omega)|^{2}=\frac{1}{1+(\Omega / \omega)^{2 n}} \text { Filtro Pasaaltas, }
\end{aligned}
$$

donde $n$ es el número de polos que se emplean para aproximar el espectro de potencia del filtro, $\omega$ es la frecuencia angular y $\Omega$ es la frecuencia de corte, donde el espectro de potencia se atenúa a la mitad de su valor máximo, o bien, donde la amplitud decrece a la raíz cuadrada de la amplitud máxima. Los respectivos homólogos en el caso de los filtros de echado son

$$
\begin{aligned}
& |H(\omega, k)|^{2}=\frac{1}{1+(D \omega / k)^{2 n}} \text { Filtro Pasaaltos, } \\
& |H(\omega, k)|^{2}=\frac{1}{1+(k / D \omega)^{2 n}} \text { Filtro Pasabajos, }
\end{aligned}
$$

donde D es la inclinación de corte, en la que la potencia del espectro se reduce a la mitad y su relación con el parámetro $\rho$ es

$$
D=\rho(\Delta t / \Delta x),
$$

donde $\Delta t$ y $\Delta x$ son los intervalos de muestreo en tiempo y distancia, respectivamente.

La aplicación del filtro requiere del espectro de amplitud $|H(\omega)|$. Si separamos el filtro en un producto de factores conjugados, obtenemos

$$
\begin{gathered}
|H(\omega)|^{2}=\frac{1}{1+(\Omega / \omega)^{2 n}}=\frac{\omega^{2 n}}{\Omega^{2 n}+\omega^{2 n}}= \\
\frac{i \omega^{n}}{\Omega^{n}+i \omega^{n}} \frac{-i \omega^{n}}{\Omega^{n}-i \omega^{n}}=\overline{H(\omega)} H(\omega),
\end{gathered}
$$

donde la testa simboliza el conjugado y $\mathrm{H}(\omega)$ queda dado por

$$
H(\omega)=\frac{-i \omega}{\Omega^{n}-i \omega^{n}} .
$$

El mismo razonamiento se aplica para el filtro pasabajas.

Hay que tener en consideración la naturaleza de los polos del filtro, debido a que se utilizaron sólo aquellos que aseguran su estabilidad, una vez discretizado por medio de la transformada bilineal. Para aplicarla, utilizamos la aproximación

$$
-i \omega=i 2 \tan (\omega / 2)=\frac{2(1-z)}{1+z},
$$

donde $z=\exp (i \omega \Delta t)$, y $\Delta t$ es el intervalo de muestreo en tiempo. Dado que el filtro debe tener fase mínima para asegurar su estabilidad, sus polos deben estar fuera del círculo unitario en el plano Z (Claerbout, 1976). Suponiendo, por sencillez, que el intervalo de muestreo es unitario, podemos definir la variable $z$ como

$$
z=e^{i \omega} .
$$

Expresando $\omega$ en sus partes real e imaginaria,

$$
\begin{gathered}
\omega=\operatorname{Re}(\omega)+\mathrm{i} \operatorname{Im}(\omega),=>z=\mathrm{e}^{i \operatorname{Re}(\omega)} e^{-\operatorname{Im}(\omega)}, \\
=>|z|=\mathrm{e}^{-I m(\omega) .}
\end{gathered}
$$

Por lo tanto, los polos fuera del círculo unitario corresponden con valores de $\omega$ tales que $\operatorname{Im}(\omega)<0$ (i.e., el semiplano inferior complejo $\omega$ ). Entonces, la expresión para los polos del filtro es

$$
v_{j}=|\Omega| \exp (-i \pi(2 j+1) / 2 n) \text {, para } j=0,1, \ldots, n-1 .
$$

La aproximación del filtro se lleva a cabo aplicando en cascada los filtros de primer orden con los polos correspondientes. Sin embargo, para fines prácticos, se utiliza sólo el primero, generando así un operador recursivo que requiere una retroalimentación. Es decir, sólo requerimos la salida anterior para calcular la salida presente. 
Por otro lado, la aproximación con un polo, aunque genera una atenuación suave, resulta útil para la reducción del fenómeno de Gibbs, que consiste en rizaduras en el espectro de amplitud del filtro. Para el diseño de filtros en frecuencia, el fenómeno ocurre al utilizar un filtro cuya banda de paso termine abruptamente en la frecuencia de corte. Un filtro así es ideal porque rechaza frecuencias exactamente a partir del valor de corte, pero en el dominio del tiempo presenta el inconveniente de atenuarse en forma muy gradual. Como es necesario truncar el filtro una vez digitalizado, despreciamos valores en tiempo de la serie de Fourier que reconstruye la señal en frecuencias, de modo que la aproximación por medio de la suma de funciones senoidales carece de suficientes términos para representar adecuadamente al filtro. Los remanentes de estas funciones senoidales explican las rizaduras (Elliot, 1987).

El primer polo del filtro está dado por

$$
\nu_{0}=|\Omega| \exp (-i \pi / 2)=-i|\Omega| .
$$

Con esta aproximación, obtenemos las siguientes expresiones para los filtros en frecuencias (Hale y Claerbout, 1983):

$$
\begin{aligned}
& H(\omega)=\frac{\Omega}{\Omega-i \omega}, \\
& H(\omega)=\frac{-i \omega}{\Omega-i \omega} .
\end{aligned}
$$

En términos del polo $v_{0}$,

$$
\begin{aligned}
& H(\omega)=\frac{-i \Omega}{-i \Omega-\omega}=\frac{v_{0}}{v_{0}-\omega}, \\
& H(\omega)=\frac{-i \omega}{\Omega-i \omega}=\frac{-\omega}{v_{0}-\omega},
\end{aligned}
$$

y aplicando la transformada bilineal obtenemos

$$
\begin{aligned}
& |\mathrm{H}(z)|=\frac{i v_{0}(1+z)}{2+i v_{0}-\left(2-i v_{0}\right) z}, \\
& |\mathrm{H}(z)|=\frac{2(1-z)}{2+i v_{0}-\left(2-i v_{0}\right) z} .
\end{aligned}
$$

Las ecuaciones (5) y (6) operan como filtros recursivos pasabajas y pasaaltas, respectivamente, con frecuencia de corte $\Omega$ que no corresponde ya con la mitad del espectro de potencia. Para ello, efectuamos un mapeo de esta frecuencia utilizando de nuevo la transformada bilineal, de modo que

$$
\begin{aligned}
& |\Omega| \rightarrow 2 \tan (|\Omega| / 2),=>v_{0}=-i 2 \tan (|\Omega| / 2), \text { para } \Omega<\pi(7) \\
& v_{0}=\infty, \text { para } \Omega>=\pi .
\end{aligned}
$$

De aquí podemos inferir la forma de los filtros de echado. Para ello hacemos

$$
\Omega=k / D \text {. }
$$

De acuerdo con la notación empleada (Hale y Claerbout, 1983), y tomando en cuenta la ecuación (7), expresamos los polos del filtro como

$$
\begin{aligned}
& v_{0}=-i \mathrm{~B}_{k}, \text { donde } \mathrm{B}_{k}=2 \tan (|\Omega| / 2) \text {, para } \Omega<\pi \\
& \mathrm{B}_{k}=\infty \text {, para } \Omega>=\pi .
\end{aligned}
$$

En términos de $B_{k}$, los filtros (5) y (6) quedan expresados como

$$
\begin{aligned}
& \mathrm{H}_{k}(z)=\frac{\mathrm{B}_{k}(1+z)}{2+\mathrm{B}_{k}-\left(2-\mathrm{B}_{k}\right) z} \text { Filtro pasaaltos, } \\
& \mathrm{H}_{k}(z)=\frac{2(1-z)}{2+\mathrm{B}_{k}-\left(2+\mathrm{B}_{k}\right) z} \text { Filtro pasabajos. }
\end{aligned}
$$

Hasta ahora hemos discretizado un filtro en $f-k$, auxiliándonos del caso similar para el dominio de $f$. Podemos pasar al dominio $t-k$ aplicando el filtro en forma recursiva a una señal digitalizada.

Desde el punto de vista del dominio $f-k$,

$$
\mathrm{Q}_{k}(\mathrm{Z})=\mathrm{H}_{k}(\mathrm{Z}) \mathrm{P}_{k}(\mathrm{Z})
$$

donde $P$ es una señal de entrada y $Q$ es la señal de salida. Utilizando las ecuaciones (9) y (10),

$$
\begin{aligned}
& \left|2+\mathrm{B}_{k}-\left(2-\mathrm{B}_{k}\right) Z\right| \mathrm{Q}_{k}(\mathrm{Z})=\mathrm{B}_{k}(1+Z) \mathrm{P}_{k}(\mathrm{Z}), \\
& \left|2+\mathrm{B}_{k}-\left(2+\mathrm{B}_{k}\right) \mathrm{Z}\right| \mathrm{Q}_{k}(\mathrm{Z})=2(1-Z) \mathrm{P}_{k}(\mathrm{Z}) .
\end{aligned}
$$


DOI: http://dx.doi.org/10.22201/fi.25940732e.2002.03n3.013

La multiplicación por un polinomio en $\mathrm{Z}$ genera retraso en el dominio del tiempo. Por lo tanto,

$$
\begin{aligned}
& \left(2+B_{k}\right) Q_{t, k}=\left(2-B_{k}\right) Q_{t-1, k}+B_{k}\left(P_{t, k}+P_{t-1, k}\right), \\
& \left(2+B_{k}\right) Q_{t, k}=\left(2-B_{k}\right) Q_{t-1, k}+2\left(P_{t, k}-P_{t-1, k}\right) .
\end{aligned}
$$

Los filtros (13) y (14) operan sobre una señal en el dominio $t-k$, y admiten que los echados varien en función del tiempo. Un razonamiento similar permite la obtención de un filtro $f-\chi$, donde puede existir variación en función de la distancia $x$. Sin embargo, el objetivo es un filtro en el dominio $t-\chi$. En las ecuaciones (13) y (14), los productos de las funciones de $k$ corresponden a convoluciones en $x$. Para el caso de las constantes, la multiplicación de una constante por una función en un dominio, implica la convolución de tal función con un impulso en el otro.

Con base en lo anterior, podemos expresar (13) y (14) como

$$
\begin{gathered}
\left(2 \delta_{x}+b_{x}\right) * a_{t, x}=\left(2 \delta_{x}-b_{x}\right) * a_{t-1, x} \\
+b_{x}^{*}\left(p_{t, x}+p_{t-1, x}\right) \\
\left(2 \delta_{x}+b_{x}\right) * a_{t, x}=\left(2 \delta_{x}-b_{x}\right) * a_{t-1, x} \\
+2\left(p_{t, x}-p_{t-1, x}\right)
\end{gathered}
$$

donde $b_{x}$ es la transformada inversa discreta de $B_{k}$.

El lector recordará que esta última procede de la transformada bilineal de la relación k/D (ecuación 8). Se puede aproximar la función tangente utilizando su serie de Taylor alrededor de $k / D=0$. Así,

$$
\tan |k /(2 D)|=\tan (0)+|k /(2 D)| \sec ^{2}(0)+\ldots
$$

Truncando hasta el segundo término, y como el primero es nulo, al sustituir en la ecuación (8) obtenemos

$$
B(k)=2|k /(2 D)|=|k / D| .
$$

Haciendo la transformada inversa de Fourier para número de onda menor al de Nyquist, y con un intervalo de muestreo $\Delta x$ unitario,

$$
\begin{gathered}
b(x)=(\pi / \mathrm{D}) \mid \operatorname{sen}(\pi x) /(\pi x) \\
-(1 / 2) \operatorname{sen}^{2}(\pi x / 2) /(\pi x / 2)^{2} \mid .
\end{gathered}
$$

La discretización de $b(x)$ requiere de un truncamiento. Para $|x|=1$ sólo obtenemos tres coeficientes, cuyos valores son (Hale y Claerbout, 1983)

$$
\boldsymbol{a}=|-2 /(\pi \mathrm{D}), \pi /(2 \mathrm{D}),-2 /(\pi \mathrm{D})|=\left|a_{-1}, a_{0}, a_{1}\right| .
$$

Como es posible expresar la convolución como una operación matricial (Claerbout, 1993), las expresiones (15) y (16) quedan en forma de productos matriciales con vectores

$$
\begin{aligned}
& (2 \mathrm{I}+\mathrm{A}) \boldsymbol{q}_{\boldsymbol{t}}=(2 \mathrm{I}-\mathrm{A}) \boldsymbol{q}_{\boldsymbol{t}-1}+\mathrm{A}\left(\boldsymbol{p}_{\boldsymbol{t}}+\boldsymbol{p}_{t-1}\right), \\
& (2 \mathrm{I}+\mathrm{A}) \boldsymbol{q}_{\boldsymbol{t}}=(2 \mathrm{I}-\mathrm{A}) \boldsymbol{q}_{\boldsymbol{t}-1}+2\left(\boldsymbol{p}_{t}-\boldsymbol{p}_{t-1}\right),
\end{aligned}
$$

donde I es la matriz identidad, y la matriz cuadrada A contiene los elementos del vector $\boldsymbol{a}$, de manera que la diagonal principal consiste del elemento $a_{0}$, le precede en el renglón $a_{-1}$ y le sucede $a_{1}$. Los demás elementos son cero, de modo que la matriz es tridiagonal.

El programa de cómputo que utilizamos para las aplicaciones de la siguiente sección se basa en las ecuaciones que se acaban de analizar. Está escrito en lenguaje $C$ y fue adaptado de una versión original de Dave Hale de la Universidad de Stanford, EUA.

\section{Aplicación a datos sísmicos de exploración}

A continuación presentamos la aplicación de los filtros de echado recursivos en dos problemas típicos en sismología de exploración. Utilizamos dos secciones. La primera es un registro de datos de campo someros, llevado a cabo en un valle aluvial cercano al río Thames, Inglaterra (Baker, 1999). La segunda es una sección marina apilada, que fue migrada en tiempo (i.e., enfocada), proveniente de la costa del Pacífico en el Sur de California, EUA, alrededor de la zona de falla de Hosgri (Honjas et al., 1992; Honjas, 1993; Pullammanappallil y Louie, 1994). 
En la primera sección, el objetivo es atenuar lo más posible la onda de aire, caracterizada como la señal con echado más pronunciado (Figura 5a). Asimismo, se busca resaltar las reflexiones horizontales que resultan afectadas por ruido en el sector izquierdo de la sección.

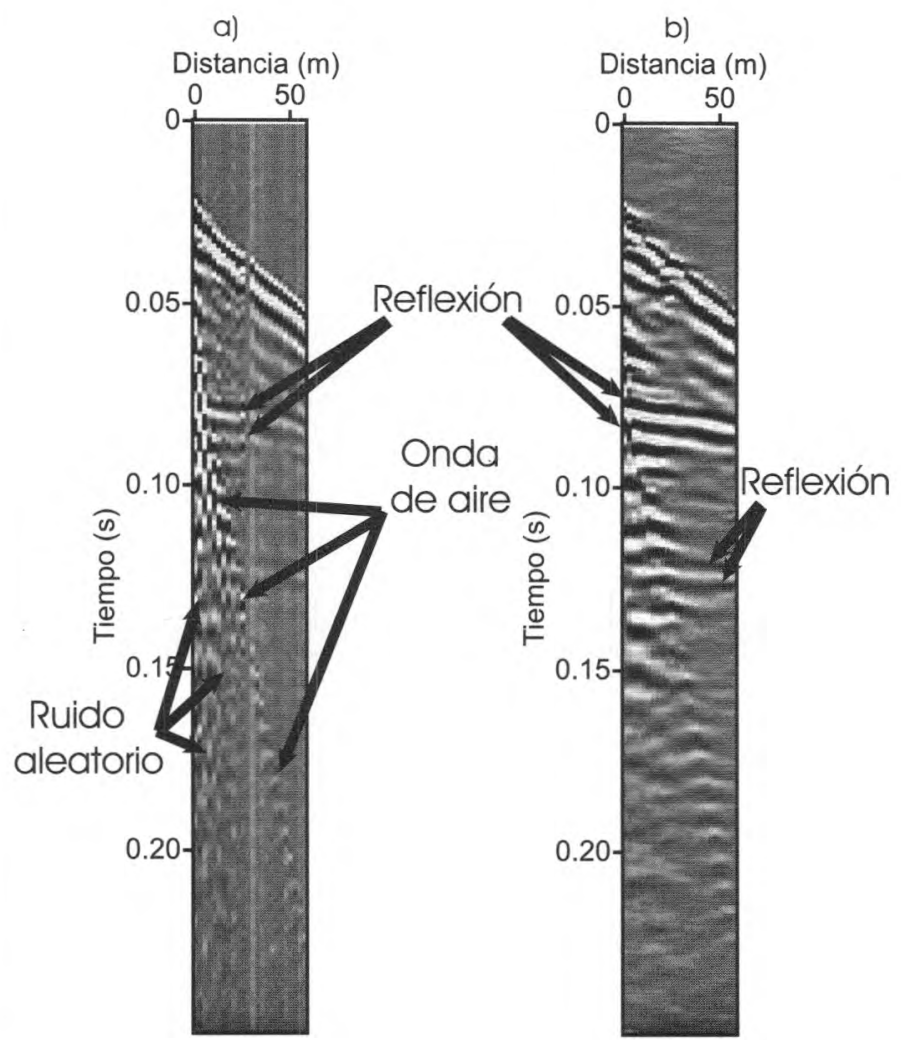

Figura 5. a) Registro de campo somero formado por 24 trazas con intervalo de muestreo de $0.5 \mathrm{~ms}$, perteneciente a un registro de tiro común cercano al río Thames, Inglaterra (Baker, 1999). La onda de aire y el ruido aleatorio obstruyen las reflexiones. 6) Sección anterior después de aplicarle un filtrado de echado pasabajos, con parámetros $\rho=10^{-7} y f_{n}=0.5$. Ahora es más sencillo identificar las reflexiones alrededor de 0.07 y $0.12 \mathrm{~s}$

En la figura 5b está el producto del filtrado de la figura $5 \mathrm{a}$. La onda de aire se ha atenuado. Sus remanentes son segmentos horizontales. El ruido que opacaba la señal en el sector izquierdo casi ha desaparecido. No obstante, surgen también problemas como la distorsión de algunas señales cuya inclinación es intermedia, como en la parte superior de la sección. Por otro lado, un filtrado con una banda de paso excesivamente reducida enfatiza artefactos horizontales irrelevantes, que pueden ser confundidos con señales genuinas.

En la segunda sección (Figura 6) empleamos, en primer lugar, un filtrado pasabajos para mostrar la atenuación de las señales asociadas con la falla, y que se encuentran casi a la mitad de la sección Posteriormente, un filtrado pasaaltos resaltará tales eventos, atenuando la estratificación.

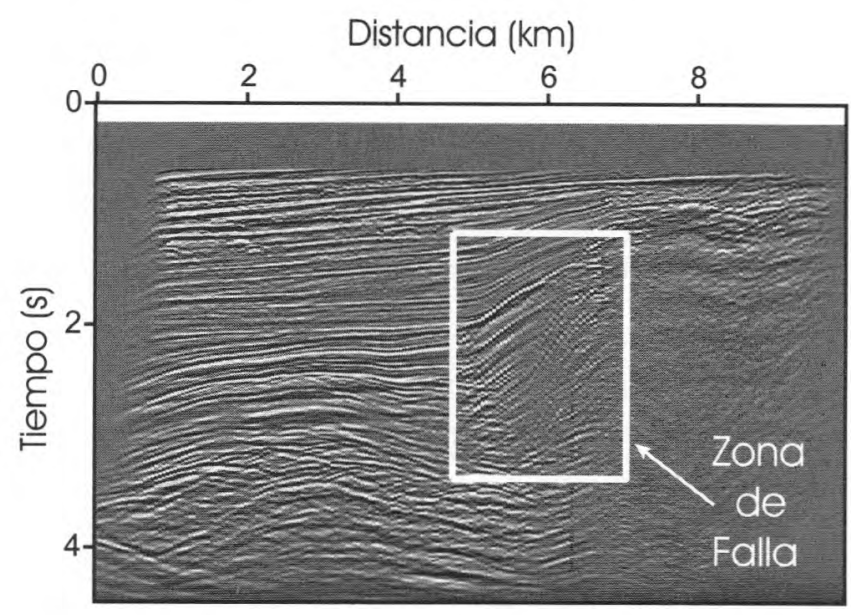

Figura 6. Sección marina proveniente de la costa del Pacífico, en el sur de California, alrededor de la falla de Hosgri (Honjas et al., 1992). La sección fue migrada en tiempo después de apilar. La zona de falla está indicada dentro del rectángulo, en la cual los eventos inclinados exhiben traslape (aliasing) espacial. Se aprecia el contacto de dos litologías diferentes, una de ellas estratificada y la otra masiva

\section{Discusión}

En la sección marina, las señales asociadas con la falla se han atenuado en su mayor parte por el filtrado pasabajos (Figura 7). Los remanentes de estas señales se deben a que el filtro deja pasar parte del espectro que les corresponde, con una amplitud menor. En el resto de la sección se han suavizado los rasgos estructurales, incluso en el sector izquierdo, entre 3 y 4 segundos, donde la geología es bastante compleja. En el sector derecho de la sección, la estratificación está ausente, a excepción de las capas superiores. Por lo 
DOI: http://dx.doi.org/10.22201/fi.25940732e.2002.03n3.013

tanto, la sección filtrada ayuda a confirmar un contacto de dos litologías diferentes. La roca a la derecha de la falla es distinta a la que se encuentra estratificada.

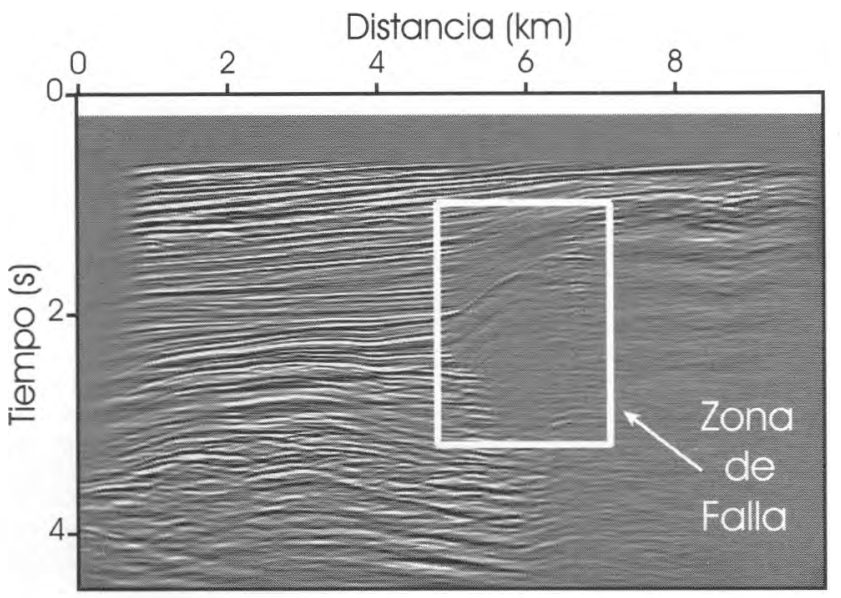

Figura 7. Sección marina después de un filtrado de echado pasabajos, con parámetros $\rho=10^{-7}$ y $f_{n}=0.5$. La estratificación ha sido enfatizada. Los eventos asociados con la falla han sido atenuados, así como los detalles estructurales en la mitad izquierda, entre 3 y $4 \mathrm{~s}$. La zona de falla está indicada dentro del rectángulo

En el caso del filtrado pasaaltos (Figura 8), hemos eliminado prácticamente todos los echados horizontales. Resaltan las señales asociadas con la zona de transición hacia la falla en la región central de la sección. Inclusive se observa el fenómeno de traslape espacial que afecta a estas señales, y que crea la impresión de que las capas buzan en dirección opuesta. No obstante, este problema no afecta al proceso de filtrado. En la parte inferior de la sección surgieron algunos artefactos numéricos de la migración. También en este caso se distinguen las dos litologías.

En ambos casos, no se observan efectos de borde (distorsión que se debe a la ausencia de datos en los límites de la imagen), que efectivamente no existen en este tipo de filtrado. Por otro lado, la ejecución del filtrado sobre estas secciones presentó inevitablemente el inconveniente de la determinación del valor de corte óptimo por medio de ensayo y error

En lo referente a otras técnicas de los filtros de echado, recientemente ha comenzado a utilizarse una variante denominada "filtrado de mediana" (de
Groot-Bril Earth Sciences BV, 2000), que opera en forma similar a los filtros de promedio móvil (moving average), pero en lugar de sustituir el valor de amplitud de una muestra por el promedio de las que se encuentran alrededor, lo sustituye por la mediana estadística. Este filtro se utiliza en dos técnicas diferentes, una para la eliminación de ruido coherente, y otra para ruido incoherente.

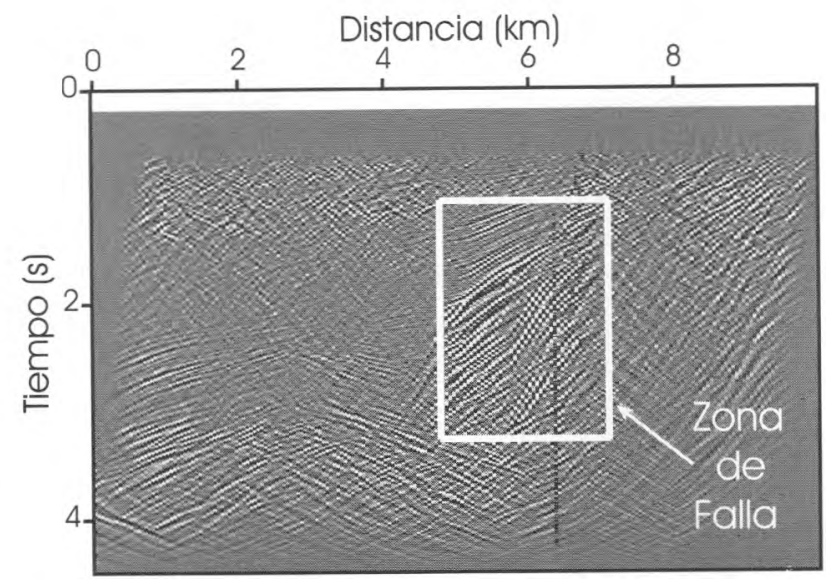

Figura 8. Sección marina después de un filtrado de echado pasaaltos, con parámetros $\rho=3 y f_{n}=0.5$. La estratificación ha sido atenuada. Destacan ahora los eventos inclinados alrededor de la falla, así como detalles estructurales adicionales. La zona de falla está indicada dentro del rectángulo. El fenómeno de submuestreo (aliasing) es más claro que en las figuras anteriores y crea la ilusión de que las capas buzan en dirección opuesta

La técnica de eliminación de ruido coherente consiste en realizar un filtrado en el que únicamente éste pasa, mientras que la señal sísmica y otros tipos de ruidos no. Finalmente, a la sección original se le resta la filtrada. El problema de este tipo de filtrado, es que en su primera etapa, no rechaza totalmente la señal sísmica, de modo que perdemos información durante la sustracción. La determinación del ángulo variable de un evento hacia el que se realizará el filtrado se determina con base en un análisis automático de semblanza (Duncan y Beresford, 1995).

Para eliminar ruido incoherente, aprovechamos que la respuesta al impulso unitario del filtro de mediana es nula (Duncan y Beresford, 1995). Así, se atenúa el ruido constituido por impulsos. El filtro permite el paso de eventos coherentes, que 
suponemos son las señales sísmicas. Este tipo de método ya comienza a incluirse en paqueterías de cómputo comercial en la industria del petróleo (e.g., de Groot-Bril Earth Sciences BV, 2000).

En la figura 9 mostramos un ejemplo de este tipo de filtrado, aplicado a una sección proveniente de las costas europeas. Antes del filtrado, la sección presenta una gran cantidad de ruido aleatorio, mismo que dificulta la interpretación de los detalles estructurales en la parte inferior de la sección, así como en los plegamientos más someros. Después del filtrado, resulta más sencillo identificar los rasgos estructurales, sin detrimento de los eventos horizontales o inclinados.
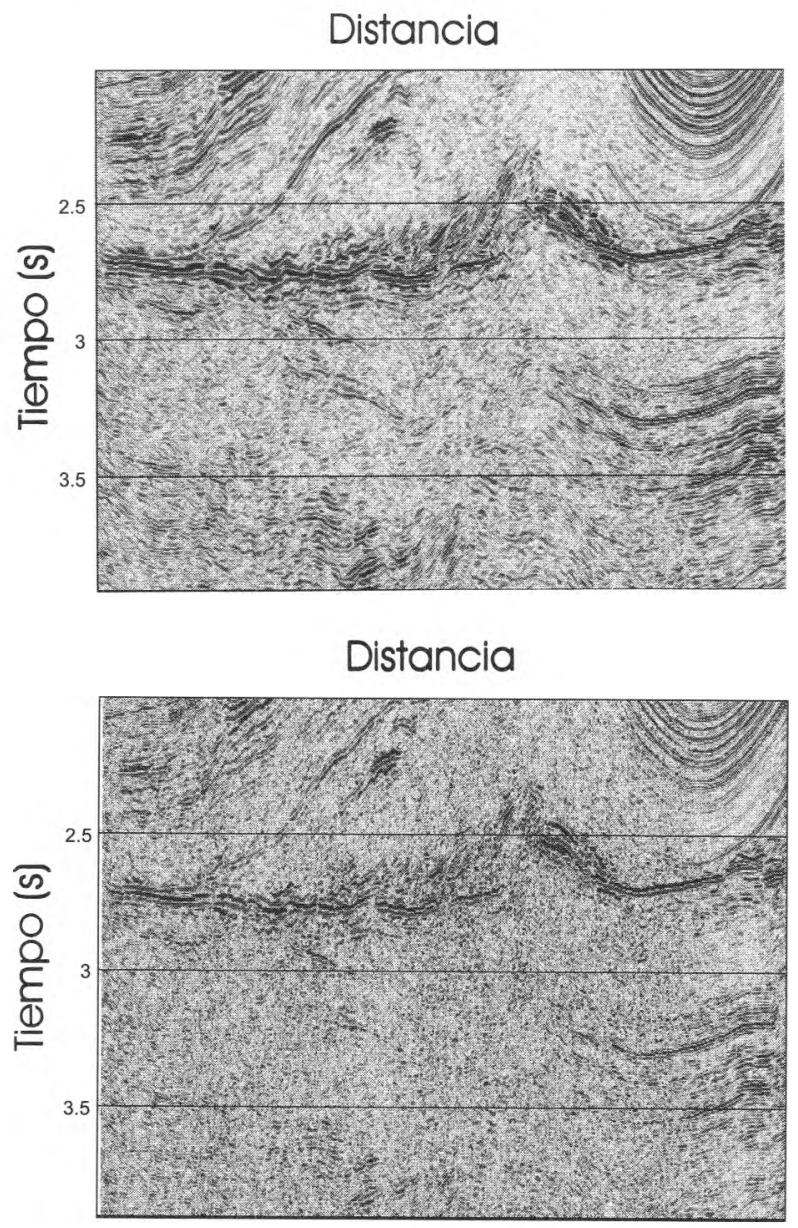

Figura 9. a) Sección sísmica antes de la aplicación de un filtro de mediana. El ruido incoherente obstruye la visualización de los detalles estructurales más finos. 6)

Sección anterior después de aplicarle un filtro de mediana. El ruido aleatorio ha sido eliminado por constar, en su mayor parte, de impulsos aislados. (Tomada del URL http://www.dg6.nl)
Los filtros de mediana eliminan ruido coherente e incoherente con eficacia, pero su uso está restringido a paqueterías de cómputo comercial. Las consideraciones con respecto al análisis de semblanza que se requiere hacen difícil implantarlo como herramienta casera.

\section{Conclusiones}

Los filtros de echado recursivos en tiempo, permiten discriminar valores de echado en forma práctica, tomando en cuenta las variaciones espaciales y temporales del echado, a diferencia de los filtrados donde transformamos de acuerdo con Fourier alguna de las dimensiones, o ambas. La aproximación con un polo suaviza la región de corte del filtro para evitar el fenómeno de Gibbs. Por otro lado, la atenuación tan suave origina problemas para elegir un valor adecuado para la banda de paso del filtro, dando lugar a que se tenga que recurrir al ensayo y error para seleccionarla. Incluso encontrando un valor aparentemente idóneo, los eventos cuya inclinación esté en las inmediaciones de la banda de rechazo sufren distorsiones

Los datos sísmicos utilizados demuestran que estos filtros trabajan adecuadamente para enfatizar imágenes, sin que el fenómeno de submuestreo afecte su desempeño.

\section{Referencias}

Baker G.S. (1999). Processing Near-Surface Seismic-Reflection Data: a Primer. Society of Exploration Geophysicists, Tulsa, OK.

Claerbout I.F. (1976). Fundamentals of Geophysical Data Processing. Mc Graw-Hill, New York. IDisponible en forma electrónica en el URL: http://sepwww.stanford.edu/sep/prof/index.htmll. Claerbout J.F. (1992). Earth Sounding Analysis: Processing Versus Inversion. Blackwell Scientific Publications. [Primeras 100 páginas disponibles en el URL http://sepwww.stanford.edu/sep/prof/index.htmll.

Claerbout J.F. (1993). Basic Earth Imaging. IVersión electrónica disponible en el URL http://sepwww.stanford.edu/sep/prof/index.htmll. 
DOI: http://dx.doi.org/10.22201/fi.25940732e.2002.03n3.013

de Groot-Bril Earth Sciences BV (2000). dGB++ Newsletter of dGB. Boletín de febrero. IURL: http://www.dgb.nll.

Duncan G. y Beresford G. (1995). Median Filter Behavior with Seismic Data. Geophysical Prospecting, Vol. 43, 329-345.

Elliot D.F. (1987). Handbook of Digital Signal Processing: Engineering Applications. Academic Press, Inc., San Diego, California.

Hale D. y Claerbout J.F. (1983). Butterworth Dip Filters. Geophysics, Vol. 48, 1033-1038.

Honjas, B., Louie, J. y Pullammanappallil S. (1992).

Results of Post and Prestack Migrations Imaging the Hosgri Fault, Offshore Santa Maria Basin, CA. EOS Supplement, Vol. 73, 390.
Honjas W.J. (1993). Results of Post- and Pre-Stack Migrations: Imaging the Hosgri Fault, Offshore Santa Maria basin, CA. Tesis de maestría, University of Nevada, Reno, NV, EUA.

Pullammanappallil S.K. y Louie J.N. (1994). A Generalized Simulated-Annealing Optimization for Inversion of First-Arrival Times. Bulletin of the Seismological Society of America, Vol. 84, 1397-1409.

Spitz S. (1993). Adaptative Dip Filtering in the $f-k$ Domain. Sixty-Third Annual Meeting and International Exposition, Society of Exploration Geophysicists. September 26-30, 1181-1182.

Yilmaz Ö. (2000). Seismic Data Analysis, Processing, Inversion and Interpretation of Seismic Data Vol. 1. Society of Exploration Geophysicists, Tulsa, OK.

\section{Semblanza de los autores}

Alejandro Cabrales-Vargas. Obtuvo el título de ingeniero geofísico en la Facultad de Ingeniería de la UNAM en enero de 2002. Su área de interés profesional es la sismología de exploración. En particular, los procesos de deconvolución y representación sísmica.

Sergio Chávez-Pérez. Obtuvo los títulos de ingeniero geofísico en 1984 en la Facultad de Ingeniería de la UNAM, de maestro en ciencias (geofísica) en 1987 en la Universidad de Carolina del Sur, EUA y de doctor en filosofía (geofísica) en 1997 en la Universidad de Nevada, Reno, EUA. Actualmente es investigador en el Instituto Mexicano del Petróleo, profesor de asignatura en la Facultad de Ingeniería de la UNAM y miembro del Sistema Nacional de Investigadores. Sus áreas de interés profesional son: representación sísmica (migración, modelado e inversión), procesamiento de datos y transformación de campos de ondas, interpretación de datos sísmicos y propagación de ondas sísmicas. 